\title{
Preemptive analgesia for postoperative pain relief in thoracolumbosacral spine operations: a double-blind, placebo-controlled randomized trial
}

\author{
Linda S. Aglio, MD, MS, ${ }^{1}$ Muhammad M. Abd-El-Barr, MD, PhD, ${ }^{2}$ Vwaire Orhurhu, MD, MPH, ${ }^{4}$ \\ Grace Y. Kim, MD, ${ }^{1}$ Jie Zhou, MD, MS, MBA, ${ }^{1}$ Laverne D. Gugino, MD, PhD, ${ }^{1}$ Lisa J. Crossley, MD, ${ }^{1}$ \\ James L. Gosnell, RN, BSN, ${ }^{1}$ John H. Chi, MD, MPH, ${ }^{3}$ and Michael W. Groff, MD ${ }^{3}$ \\ Departments of ${ }^{1}$ Anesthesiology, Perioperative and Pain Medicine, and ${ }^{3}$ Neurosurgery, Brigham \& Women's Hospital, Harvard \\ Medical School, Boston, Massachusetts; ${ }^{2}$ Department of Neurosurgery, Duke University Medical Center, Durham, North \\ Carolina; and ${ }^{4}$ Department of Anesthesia, Critical Care and Pain Medicine, Beth Israel Deaconess Hospital, Harvard Medical \\ School, Boston, Massachusetts
}

OBJECTIVE Preemptive administration of analgesic medication is more effective than medication given after the onset of the painful stimulus. The efficacy of preoperative or preemptive pain relief after thoracolumbosacral spine surgery has not been well studied. The present study was a double-blind, placebo-controlled randomized trial of preemptive analgesia with a single-shot epidural injection in adult patients undergoing spine surgery.

METHODS Ninety-nine adult patients undergoing thoracolumbosacral operations via a posterior approach were randomized to receive a single shot of either epidural placebo (group 1), hydromorphone alone (group 2), or bupivacaine with hydromorphone (group 3) before surgery at the preoperative holding area. The primary outcome was the presence of opioid sparing and rescue time-defined as the time interval from when a patient was extubated to the time pain medication was first demanded during the postoperative period. Secondary outcomes include length of stay at the postanesthesia care unit (PACU), pain score at the PACU, opioid dose, and hospital length of stay.

RESULTS Of the 99 patients, 32 were randomized to the epidural placebo group, 33 to the hydromorphone-alone group, and 34 to the bupivacaine with hydromorphone group. No significant difference was seen across the demographics and surgical complexities for all 3 groups. Compared to the control group, opioid sparing was significantly higher in group $2(57.6 \%$ vs $15.6 \%, p=0.0007)$ and group $3(52.9 \%$ vs $15.6 \%, p=0.0045)$ in the first demand of intravenous hydromorphone as a supplemental analgesic medication. Compared to placebo, the rescue time was significantly higher in group 2 (187 minutes vs 51.5 minutes, $p=0.0014)$ and group 3 (204.5 minutes vs 51 . minutes, $p=0.0045)$. There were no significant differences in secondary outcomes.

CONCLUSIONS The authors' study demonstrated that preemptive analgesia in thoracolumbosacral surgeries can significantly reduce analgesia requirements in the immediate postoperative period as evidenced by reduced request for opioid medication in both analgesia study groups who received a preoperative analgesic epidural. Nonetheless, the lack of differences in pain score and opioid dose at the PACU brings into question the role of preemptive epidural opioids in spine surgery patients. Further work is necessary to investigate the long-term effectiveness of preemptive epidural opioids and their role in pain reduction and patient satisfaction.

Clinical trial registration no.: NCT02968862 (clinicaltrials.gov)

https://thejns.org/doi/abs/10.3171/2018.5.SPINE171380

KEYWORDS preemptive analgesia; spine surgery; postoperative pain; analgesia; lumbar; thoracic; sacral

$\mathrm{T}$ HERE have been many studies that suggest that any noxious stimulus, including a surgical incision, may induce prolonged changes in the central nervous function. These changes may contribute to postoperative pain. Various methods have been used to decrease post- operative pain, which has been shown to decrease patient satisfaction and decrease hospitalization stays and complications. ${ }^{20}$

Preemptive analgesia, which is defined as the preoperative administration of analgesia prior to the painful stim-

ABBREVIATIONS LOS = length of stay; PACU = postanesthesia care unit; VAS = visual analog scale.

SUBMITTED December 18, 2017. ACCEPTED May 11, 2018.

INCLUDE WHEN CITING Published online September 14, 2018; DOI: 10.3171/2018.5.SPINE171380. 
ulus, has shown promise in spine surgery, with various methods and drugs having been used. ${ }^{6}$ Epidural preemptive analgesia has shown some promise in thoracolumbosacral surgery. ${ }^{13,18}$ The most common agents in these epidural injections have been local anesthetic agents. ${ }^{13,18}$ The mechanism of action of these local anesthetics is to block neural transmission of pain signal and/or desensitize local nociceptors, both effective measures to decrease postoperative pain perception. ${ }^{13}$ The most common disadvantages of using these local anesthetics are the possibility of transient motor blockade and indwelling epidural catheter problems. ${ }^{6,22}$

Neuroplasticity induced by the painful stimulus can be prevented or preempted by the administration of analgesics prior to injury or pretreatment with $\mu$-opioid agonist receptors.7 Some of the advantages of using epidural opioids is that the degree of postoperative analgesia is superior to that achieved by intramuscular administration or intravenous patient-controlled analgesia (PCA). ${ }^{2,9}$

Some studies of epidural opioids have shown an additive and improved effect with local anesthetics. Others have shown no improvement in postoperative pain scores with a considerable risk of negative consequences, including spinal epidural abscess, hypotension, and respiratory depression..$^{22,25}$ The data are lacking in attempting to prove that epidural analgesia is effective in providing postoperative analgesia after thoracolumbosacral spine surgery.

We designed a double-blind, placebo-controlled randomized trial to study the effects of a single dose of preoperative epidural hydromorphone or bupivacaine with hydromorphone compared to a placebo on postoperative pain and analgesic requirements. The population of patients was undergoing elective thoracolumbosacral spine surgery at a tertiary academic surgical center. The primary outcome was the incidence of opioid sparing and rescue time-defined as the time interval from when a patient was extubated to the time pain medication was first demanded in the postoperative period. Secondary outcomes were amount of intravenous hydromorphone used at the postanesthesia care unit (PACU), visual analog scale (VAS) score on rescue, hospital length of stay (LOS), and total oxycodone amounts on postoperative days 0,1 , and 2 .

\section{Methods}

The study was performed under the supervision of the Partners Health Care and Brigham and Women's Internal Review Board. In addition, the protocol was registered with clinicaltrials.gov on November 18, 2016, with unique identifier NCT02968862. Adult patients who were seen at Brigham and Women's Hospital, Department of Neurosurgery, for elective thoracolumbosacral spine surgery were prospectively enrolled in the study during their routine preoperative visit in 2014-2016. To be eligible for enrollment, patients needed to be relatively healthy, consistent with inclusion and exclusion criteria, and have an American Society of Anesthesia (ASA) grade of 2 or better. Patients undergoing either new or repeat decompressions or fusions of the spine were included in this study. We did not exclude patients with psychological comorbidities; however, patients with extensive preoperative pain medication requirements based on their history prior to surgery and medical records were excluded, especially those with extensive narcotic used. Extensive pain medication use was defined by the use of medication in two or more of the following medication categories: neuropathics, opioids, or muscle relaxants. Patients with spine tumors, spinal cord tumors, or infections were excluded. Written informed consent was obtained from all subjects or a legal surrogate.

The sample size was calculated to obtain a power of $85 \%$ at an alpha level of $5 \%$.

The power calculation for the study was based on the time it took for a patient to request analgesia after surgery. We assumed a $25 \%$ increase in time for patients requesting analgesia in both drug groups compared to the placebo in the PACU. To detect this increase in time after surgery, 32 subjects per treatment arm would be needed for a study with an alpha level of 0.05 (two-tailed test) and a beta level of 0.2 (80\% power). ${ }^{3}$

Ninety-nine patients were randomized to 1 of 3 groups of equal size, using a publicly available program (www. randomization.com), using block sizes of 3. Pharmacy generated the randomization schema containing 150 treatments numbered sequentially $1-150$. The treatments were grouped in blocks of 3 . Pharmacy randomized subjects sequentially on the morning of surgery. The randomization sequence was maintained in a restricted area to which study staff did not have access. The final product was blinded to all individuals involved in the study. Group 1 received an epidural with $10 \mathrm{ml}$ of normal saline (placebo). Group 2 received an epidural analgesic that consisted of $0.5 \mathrm{mg}$ of hydromorphone in $10 \mathrm{ml}$ of normal saline. Group 3 received an epidural analgesic that consisted of $31.25 \mathrm{mg}$ of bupivacaine and $0.5 \mathrm{mg}$ of hydromorphone in $10 \mathrm{ml}$ of normal saline. The doses for the preemptive analgesia were based on previous studies ${ }^{9,24}$ and our own clinical experience. It is important that the dose be high enough to provide postoperative analgesia but low enough as to allow the possibility of intraoperative neurophysiological monitoring, which included motor and sensory somatosensory evoked potentials. Patients were prospectively studied in a double-blind manner. Randomization and blinding were done by the pharmacy at the Brigham and Women's Hospital. Epidural administration was performed in a standard fashion either by an anesthesiology resident supervised by a board-certified anesthesiologist or solely by a board-certified anesthesiologist. In this standard approach, patients were asked to sit upright, and the interlaminar space between the L2 and L5 vertebrae was independently selected by the operator. The Tuohy needle was inserted midline or paramedian until the epidural space was identified with air or normal saline. The epidural medications were subsequently administered at the epidural space. Signs of local anesthetic toxicity, intravascular injection, intrathecal injections, spinal headache, and post-dural puncture headache were monitored. There were no reported single-shot epidural-related complications after each procedure.

Primary outcome was rescue time, defined as the time interval from when the patient was extubated to the time pain medication was first administered during the post- 
operative period and presence of opioid sparing (proportion of patients who did not ask for opioid medication in the PACU). Secondary outcomes included the amount of intravenous hydromorphone used at the PACU), the VAS score on rescue, hospital LOS, and total oxycodone amounts on postoperative days 0,1 , and 2 . Other secondary outcomes included oxygen saturation and sedation score every 15 minutes for 2 hours in the PACU. Of note, the rescue drug regimen was the standard protocol for all PACU patients, with a pain score of 1 or above. This was hydromorphone IV $0.2-0.4 \mathrm{mg}$ every 5 minutes up to 10 doses. For safety purposes, we also documented any cases of intraoperative and postoperative respiratory depression that could be explained by use of hydromorphone as well as the use of any opioid-reversal agents.

Prior to any intervention, patients were familiarized with the use of the 10-cm VAS and baseline VAS scores were noted in the preoperative holding area. Epidural catheters were placed one or two interspaces above the site of surgery preoperatively in the holding area at least 1 hour prior to the surgical incision by one of our anesthesiologists. The accuracy of the epidural needle placement was determined by using standard anatomical landmarks and palpating the patient's anatomy. No additional analgesics were used preoperatively except $2-3 \mathrm{ml}$ of $1 \%$ lidocaine in the skin where the epidural was placed.

Anesthesia was induced with propofol $(1.5-2.5 \mathrm{mg} / \mathrm{kg})$. Maintenance consisted of oxygen in air with total intravenous anesthesia of propofol and remifentanil with a hydromorphone bolus on a weight-based scale. Hydromorphone was administered at the end of the surgical case before patients were transported to the PACU.

Surgical technique was categorized as simple or complex. A simple surgery was defined as decompression surgery involving one lumbar vertebral segment without fusion of the spine. The complex surgical category was defined as cases with either a decompression involving more than one vertebral segment or any decompression that included fusion.

Opioid demand was defined as the first request from the patient for supplemental analgesia in the PACU. The patients were assessed every 15 minutes for the first 2 hours following surgery by a registered nurse using the VAS.

\section{Statistical Analysis}

Normality of our data distribution was determined using the Kolmogorov-Smirnov test with Lilliefors correction (StataCorp 2016, Stata: Release 12, Statistical Software). Data were analyzed by investigators not involved in the perioperative care or data collection phase of the study. Parametric data are presented as mean \pm standard deviation or as a number (percentage). We report nonparametric data as median with interquartile range as indicated in the table footnotes. For data with normal distribution, single comparisons were tested using the t-test, whereas multiple comparisons were tested with ANOVA with Tukey post hoc pairwise testing, when appropriate. A series of $3 \times 2$ chi-square tests were used to determine differences among the 3 groups, followed by $2 \times 2$ chi-square tests for intergroup differences. Follow-up analysis was corrected using the Bonferroni adjustments. The Kruskal-Wallis H- test was used to analyze nonparametric data, and multiple pairwise comparisons were performed using the MannWhitney U-test. Nominal data were analyzed by using either chi-square or the Fisher's exact test, as appropriate. $p$ $<0.05$ was considered significant.

This manuscript adheres to the applicable Equator guidelines.

\section{Results}

A total of 99 patients were evaluated in this study. No significant difference was seen in the demographics and surgical complexity across all 3 groups (Table 1). The average age of our patients was $56 \pm 13$ years. The average preoperative VAS score for all patients was $4.0 \pm 2.5$, with no significant differences among the 3 groups (Table 1). The median time between epidural placement and the start of the procedure was $62 \pm 19$ minutes, with no significant difference among all 3 groups. There were no significant differences in the type and doses of maintenance medications used across groups (Table 1). Similarly, there were no significant differences in the operative time (Table 1).

Both the epidural hydromorphone-only (group 2) and the epidural hydromorphone and bupivacaine (group 3) groups had significantly higher percentages of patients who did not require opioid rescue in the PACU $(63.6 \%$ vs $21.9 \%$, p < 0.05 , and $55.9 \%$ vs $21.9 \%$, p < 0.05 , respectively). Of note, when breaking down the results in terms of patients who had simple (one-segment) decompressive surgery compared to more complex surgery ( $\geq 2$ segments with or without fusion), some interesting characteristics were seen. The percentage of patients who did not require opioids in the PACU for decompressive surgeries in the placebo group was $23 \%(6 / 26)$, while in the hydromorphone group it was 66\% $(19 / 29)(\mathrm{p}<0.05)$ and in the hydromorphone and bupivacaine group it was 59\% (17/29) (p $<0.05)$. There was a similar trend in the group of patients who underwent fusion surgeries, but due to the small numbers, this was not statistically significant. There was no significant difference between those who received epidural hydromorphone only (group 2) and those who received the epidural hydromorphone and bupivacaine (group 3) in terms of patients not requiring opioid rescue in the PACU (Table 2). In patients who did require opioid rescue in the PACU, group 2 patients needed rescue much later than the placebo group patients (187 minutes vs 51.5 minutes, $\mathrm{p}<$ 0.05) (Fig. 1, Table 2). This was also true of the patients who received epidural hydromorphone and bupivacaine (group 3) compared to the placebo group (204.5 minutes vs 51.5 minutes, $p<0.05$ ) (Fig. 1, Table 2). There was no difference in rescue time between the 2 epidural groups (Fig. 1, Table 2). For those patients who did not require opioids in the PACU, both the group 2 and group 3 patients needed opioids later than the placebo group patients, but this did not reach statistical significance (Table 2).

Every 15 minutes of the first 2 hours, the median pain score was significantly lower among patients who received either epidural hydromorphone only (group 2) or epidural hydromorphone and bupivacaine (group 3), compared to patients who received the placebo (group 1) (Table 2). However, all 3 study groups had very similar VAS ratings 
TABLE 1. Patient characteristics in all 3 exposure groups

\begin{tabular}{|c|c|c|c|c|}
\hline \multirow[b]{2}{*}{ Characteristic } & \multicolumn{3}{|c|}{ Patient Group } & \multirow[b]{2}{*}{ p Value } \\
\hline & Placebo $(n=32)$ & Hydromorphone $(n=33)$ & Epidural $(n=34)$ & \\
\hline $\mathrm{Age}^{*}$ & $60 \pm 12.5$ & $57 \pm 27$ & $59.5 \pm 19$ & 0.8249 \\
\hline \multicolumn{5}{|l|}{ Sex } \\
\hline Male (\%) & 59.4 & 72.7 & 47.1 & 0.1035 \\
\hline Race (no.) & & & & 0.3732 \\
\hline Caucasian & 32 & 31 & 32 & \\
\hline Black & 0 & 1 & 1 & \\
\hline Asian & 0 & 1 & 1 & \\
\hline VAS score at preop location & $4.2 \pm 4.3$ & $4.5 \pm 4.8$ & $4.3 \pm 2.8$ & 0.7498 \\
\hline \multicolumn{5}{|l|}{ Intraop opioid medications ${ }^{*}$} \\
\hline Remifentanil (mcg) & $1955 \pm 1387$ & $1654 \pm 1301$ & $1614 \pm 1010$ & 0.1993 \\
\hline Fentanyl (mcg) & $100 \pm 100$ & $100 \pm 100$ & $100 \pm 50$ & 0.7207 \\
\hline IV hydromorphone (mg) & $1.8 \pm 0.8$ & $1.5 \pm 0.8$ & $1.5 \pm 0.6$ & 0.1903 \\
\hline \multicolumn{5}{|l|}{ Complexity of spine surgery (no.) } \\
\hline Simple (decompression) & 26 & 29 & 29 & \\
\hline Complex (fusion) & 6 & 4 & 5 & \\
\hline Duration of surgery (mins) & $174 \pm 78.4$ & $159 \pm 74.3$ & $154 \pm 71.3$ & 0.5922 \\
\hline Time from exposure $\nmid$ to procedure (mins) $)^{*}$ & $62 \pm 19.5$ & $66 \pm 16$ & $59 \pm 19$ & 0.1397 \\
\hline
\end{tabular}

when they required opioid rescue (Table 2). There were also no differences among the groups in percentage of patients who had over $90 \%$ oxygen saturation on room air at 2 hours (Table 2), or at any time points (data not shown). Similarly, there were no differences in the amount of time all 3 groups spent at phase I of the PACU (Table 2).

All 3 groups were very similar in their postoperative pain medication regimen at postoperative days 0,1 , and 2 (Table 2). Similarly, there was no difference in the LOS among all 3 groups (Table 2). Subgroup analysis showed that there was no difference in LOS among all 3 groups for both simple and complex surgery types.

\section{Discussion}

In the United States, pain is ranked as one of the most common reasons for seeking medical care. ${ }^{11}$ The World Health Organization estimates that $20 \%$ of the world lives with some degree of pain on a daily basis. ${ }^{8}$ In 1931, Albert Schweitzer said, "We must all die. But that I can save him from days of torture, that is what I feel as my great and ever new privilege. Pain is a more terrible lord of mankind than even death itself."

Preemptive analgesia attempts to prevent the often altered processing of afferent input, which is thought to amplify postoperative pain. ${ }^{14}$ As early as 1913 , Crile documented the concepts of preemptive analgesia based on clinical observation. He advocated the use of regional blocks in addition to general anesthesia to prevent pain. ${ }^{5}$ Further studies performed by Woolf in 1983 examined preemptive analgesia as a method of preventing postoperative pain in animal models. Those results suggested that the surgical incision and its subsequent painful postoper- ative events prolonged changes in the dorsal horn. Hence, postoperative pain may subsequently be enhanced. ${ }^{23}$ In a nice review of both the experimental and clinical data, Kissin showed that the experimental evidence for the efficacy of preemptive analgesia is convincing, but the results of clinical studies remain controversial. ${ }^{14}$

Clinical studies have been less convincing as it is clear that preemptive analgesia must cover not only incisional pain but also the pain that is associated with inflammation..$^{14,17}$ In a large review of clinical studies of preemptive analgesia, Møiniche et al. showed that evidence for clinical preemptive analgesia is mixed. ${ }^{17}$ It is noteworthy that there was an overall improvement in pain control in 7 of 11 studies in which a single-dose epidural analgesia was the vehicle of analgesia administration. This was by far the best outcome when compared to all types of analgesic intervention.

With regard to spine surgery, perioperative pain control has become an important concern for clinicians, as many spinal surgeries are associated with intense postoperative pain. Additionally, it has been shown that improved perioperative pain is associated with a better surgical outcome, decreased hospital stays, and a decrease in chronic pain medication used by patients. ${ }^{6}$ As noted by Devin and McGirt, another reason that pain control has become important to physicians is that an increasing number of quality measurement systems focus on perioperative pain control as an important factor in patient satisfaction. ${ }^{6}$

The use of preemptive analgesia in spine surgery has been found to be promising. This has included the use of Cox-2 inhibitors, ${ }^{15}$ intravenous morphine, nonopioid NSAIDs,${ }^{19}$ and even pulsed electrical modulation. ${ }^{1}$ The placement of an epidural catheter raises the concern of un- 
TABLE 2. Univariate analysis of primary and secondary outcomes across all 3 exposure groups

\begin{tabular}{|c|c|c|c|c|c|}
\hline \multirow[b]{2}{*}{ Outcome } & \multirow{2}{*}{$\begin{array}{l}\text { Placebo } \\
(n=32)\end{array}$} & \multirow{2}{*}{$\begin{array}{l}\text { Hydromorphone } \\
\text { (group A) }(n=33)\end{array}$} & \multirow{2}{*}{$\begin{array}{l}\text { Hydromorphone + Bupivacaine } \\
\text { (group B) }(n=34)\end{array}$} & \multicolumn{2}{|c|}{$p$ Value } \\
\hline & & & & Group A vs Placebo & Group B vs Placebo \\
\hline \multicolumn{6}{|l|}{ Primary outcome } \\
\hline Opioid sparing, no. (\%) & $7(21.9)$ & $21(63.6)$ & $19(55.9)$ & 0.001 & 0.005 \\
\hline Simple (decompression) & $6 / 26(23.1)$ & $19 / 29(65.5)$ & $17 / 29(58.6)$ & 0.002 & 0.008 \\
\hline Complex (fusion) & $1 / 6(16.7)$ & $2 / 4(50.0)$ & $2 / 5(40.0)$ & 0.285 & 0.409 \\
\hline Rescue time for OSP (mins) ${ }^{*}$ & $242 \pm 226$ & $329 \pm 450$ & $515 \pm 385$ & 0.305 & 0.079 \\
\hline Time to PACU rescue (mins) & 51.5 & 187.0 & 204.5 & 0.001 & 0.004 \\
\hline \multicolumn{6}{|l|}{ PACU secondary outcomes* } \\
\hline VAS score on rescue & $6.1 \pm 3.1$ & $5.7 \pm 4.2$ & $5.9 \pm 3.0$ & 0.159 & 0.596 \\
\hline Pain score at 15 mins & $3.4 \pm 6.7$ & $2.5 \pm 4.6$ & $2.5 \pm 5.5$ & 0.014 & 0.013 \\
\hline Pain score at 30 mins & $4.1 \pm 5.4$ & $2.4 \pm 4.8$ & $3.1 \pm 4.8$ & 0.010 & 0.058 \\
\hline Pain score at 45 mins & $4.3 \pm 3.9$ & $2.6 \pm 4.5$ & $3.2 \pm 4.9$ & 0.029 & 0.047 \\
\hline Pain score at 60 mins & $4.3 \pm 3.9$ & $2.4 \pm 4.9$ & $2.6 \pm 3.9$ & 0.019 & 0.011 \\
\hline Pain score at 75 mins & $4.5 \pm 3.1$ & $2.0 \pm 4.6$ & $2.3 \pm 4.5$ & 0.002 & 0.005 \\
\hline Pain score at 90 mins & $4.8 \pm 3.1$ & $2.0 \pm 4.1$ & $2.7 \pm 3.8$ & 0.001 & 0.003 \\
\hline Pain score at 105 mins & $4.9 \pm 2.6$ & $1.6 \pm 3.6$ & $2.5 \pm 3.4$ & $<0.001$ & 0.002 \\
\hline Pain score at 120 mins & $4.4 \pm 3.1$ & $2.9 \pm 3.5$ & $2.8 \pm 3.5$ & 0.003 & 0.002 \\
\hline PACU $\mathrm{O}_{2}>90 \%$ at RA, no. (\%)†‡ & $26(35.1)$ & $25(33.8)$ & $23(31.1)$ & 0.639 & 0.089 \\
\hline Sedation score $($ mean \pm SD) $\dagger$ & $26 \pm 17$ & $26 \pm 19$ & $27 \pm 16$ & 0.643 & 0.543 \\
\hline PACU IV hydromorphone (mg) & $1.6 \pm 1.7$ & $1.2 \pm 1.4$ & $1.6 \pm 1.6$ & 0.390 & 0.989 \\
\hline Duration at PACU Phase I (mins) & $150 \pm 69$ & $120 \pm 72$ & $121 \pm 64$ & 0.358 & 0.529 \\
\hline \multicolumn{6}{|l|}{ Post-PACU secondary outcomes* } \\
\hline Total oxycodone POD 0 (mg) & $10 \pm 0$ & $10 \pm 10$ & $10 \pm 5$ & 0.191 & 0.095 \\
\hline Total oxycodone POD 1 (mg) & $25 \pm 20$ & $22.5 \pm 17.5$ & $25 \pm 30$ & 0.778 & 0.419 \\
\hline Total oxycodone POD 2 (mg) & $30 \pm 35$ & $20 \pm 15$ & $35 \pm 40$ & 0.707 & 0.354 \\
\hline LOS (days) & $1 \pm 2$ & $2 \pm 2$ & $1 \pm 1$ & 0.567 & 0.854 \\
\hline
\end{tabular}

$\mathrm{OSP}=$ opioid-sparing patient; $\mathrm{O}_{2}=$ oxygen saturation; $\mathrm{RA}=$ room air.

*Values are presented as the median \pm IQR.

$\dagger$ Data collected at $T=2$ hours in the PACU.

$\ddagger$ Patients who required supplemental oxygen to maintain oxygen saturation $>90 \%$.

predictable analgesia and drug absorption from the surgical site. The catheter may migrate or kink. A single-dose epidural analgesic introduced before the surgical incision may eliminate these problems. Sufficient time must be allowed for the analgesic to bind to the target-specific receptors.

There are only a few studies that have looked at the use of epidural analgesia for preemptive analgesia in spine surgery. Kang et al. have described the use of a single epidural injection of ropivacaine in a preoperative manner in patients undergoing posterior lumbar interbody arthrodesis. ${ }^{13}$ They reported that patients who received an epidural injection of $0.1 \%$ ropivacaine 20 minutes before incision had better immediate pain control than those who received only epidural saline. Sekar et al. also reported better immediate pain control in patients who received a single epidural dose of bupivacaine and tramadol. ${ }^{18} \mathrm{In}$ an interesting study, Yukawa et al. investigated 3 different types of preoperative analgesia in patients undergoing spine surgery: continuous subcutaneous morphine, continuous epidural morphine, and diclofenac sodium. ${ }^{25}$ Interestingly, they found that the group that received continuous epidural morphine did not have better pain con- trol and had a high rate of complications (> 50\%) - mostly nausea and vomiting, although 2 patients had to have their treatment discontinued due to systemic hypotension. Also, the group that received diclofenac sodium was found to need more rescue pain medication. In a follow-up study, the same group used subcutaneous morphine and a single intrathecal dose of morphine preoperatively and found good outcomes without complications. ${ }^{24}$ Besides the hypothesized mode of action, another potential mechanism for preemptive analgesia is that intrathecal opioid administration may blunt the increased opioid tolerance seen with remifentanil infusions. ${ }^{21}$

The use of epidural hydromorphone alone or hydromorphone with bupivacaine has been used with good results in joint arthroplasty, ${ }^{12}$ scoliosis surgery,${ }^{10}$ and labor.${ }^{16}$ Both our study groups had significantly higher percentages of patients who did not require opioid rescue in the PACU. For both groups, if patients did require opioid rescue in the PACU, it was at a much later time. Interestingly, there was no significant difference between the hydromorphonealone group and the group that received both hydromorphone and bupivacaine. Some have suggested that these 


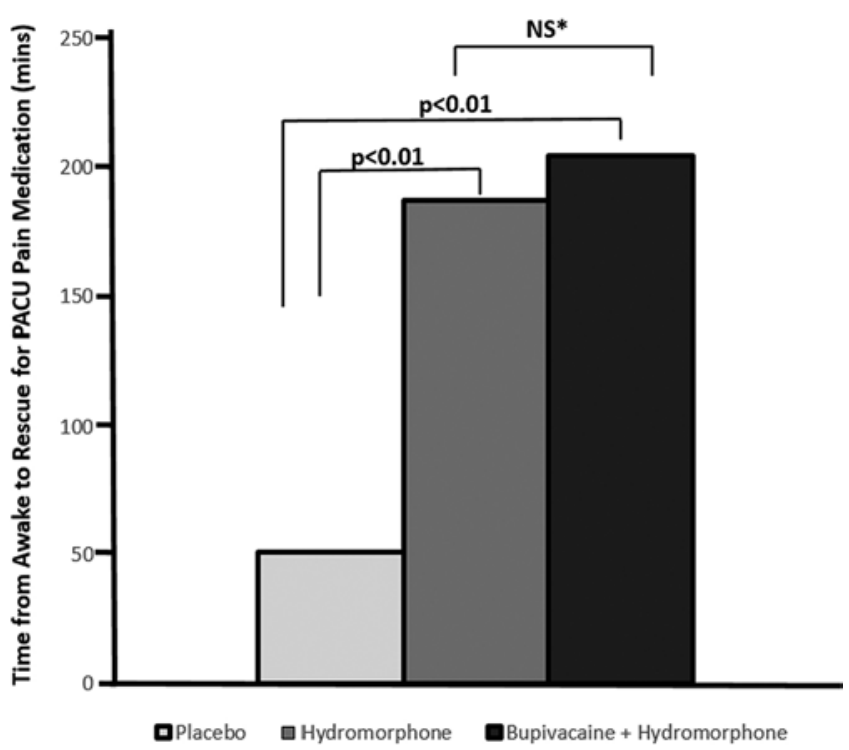

FIG. 1. Time to rescue at postoperative care unit across all 3 exposure groups. Time to rescue was significantly higher for the hydromorphone and bupivacaine/hydromorphone groups than for the control group. NS $=$ not significant.

two components may have synergistic effects, but our results do not support this. Importantly, none of our patients had evidence of the negative side effects that have been reported for epidural hydromorphone, such as systemic hypotension or respiratory distress.

Besides the immediate positive effects seen in our study groups, it is interesting to note that these study groups did not differ much from the control group in terms of their postoperative pain medication regimen in the total amount of narcotics needed in the PACU or their VAS scores. Similarly, there were no statistically significant differences in the pain regimen across all 3 groups on postoperative days 0,1 , and 2 . Also, there was no difference in the LOS among 3 groups. Does this suggest that the effect of preemptive analgesia is short lived? Bioavailability studies would suggest that hydromorphone with its hydrophilic properties binds more strongly to receptors in the dorsal horn of the spinal cord as compared to lipophilic compounds such as sufentanil. ${ }^{4}$ It may be that besides the immediate consequence of incisional pain, we may be seeing the effects of inflammation-a key component of surgical pain and one often not addressed in preemptive analgesia trials. It will be of particular interest to see how this improved patient experience in the PACU translates to overall satisfaction and long-term opioid use.

The results of this study support the data reported in prior animal studies - namely, that the spinal hyperactivity that is caused by a noxious stimulation can be prevented by prior administration of $\mu$-opioid agonist receptors. ${ }^{9}$ Furthermore, the development of central sensitization may be attenuated or prevented by preemptive analgesia. This results in a lower postoperative analgesic requirement and lower postoperative pain scores.?

This study had some limitations. First, our study design was randomized and controlled, but it did not include data like severity of disease pathology, need for osteotomy, surgical approach (minimal invasive surgery vs open vs endoscopic vs tubular), and complexity of surgical procedure. This makes it difficult to draw conclusions about complex spine fusion cases. Second, this study did not account for the psychological aspects that may contribute to chronic pain management. The average VAS score for our study cohort was low, but all patients in the 3 groups required significant pain medications. This could be explained by the heterogeneity of the spine pathologies, but we cannot rule out the contributions from other psychological comorbidities. The reduced need for pain medications immediately after surgery could be explained by heterogeneity in covariates like depression and anxiety. However, we did not specifically measure these covariates, making it difficult to draw this conclusion. The hospital and PACU LOS were not different across the groups, bringing into question the cost benefit of preemptive epidural before spine surgery

\section{Conclusions}

Our study demonstrated that preemptive analgesia in thoracolumbosacral surgeries can significantly reduce analgesia requirements in the immediate postoperative period as evidenced by reduced request for opioid in both analgesic study groups with preoperative analgesic epidural. Nonetheless, the lack of differences in pain score and opioid dose at the PACU brings into question the role of preemptive epidural opioids in spine surgery patients.

More work needs to be done to see whether this improvement in the immediate postoperative outcomes results in long-term improvement in patients' quality of life and pain control. Preemptive analgesia with a single-shot epidural injection of bupivacaine with hydromorphone or hydromorphone alone is a safe, simple, and effective method for patients undergoing thoracolumbosacral operations.

\section{Acknowledgments}

Special thanks to Patricia A. Kelly, BS, RPh, Brigham and Women's Hospital, Boston, MA, for her help with randomization and preparation of medications.

\section{References}

1. Aydoğan S, Er U, Ozlü O: Effectiveness of preemptive analgesia using a frequency rhythmic electrical modulation system in patients having instrumented fusion for lumbar stenosis. Asian Spine J 8:190-196, 2014

2. Benzon HT, Wong HY, Belavic AM Jr, Goodman I, Mitchell $\mathrm{D}$, Lefheit T, et al: A randomized double-blind comparison of epidural fentanyl infusion versus patient-controlled analgesia with morphine for postthoracotomy pain. Anesth Analg 76:316-322, 1993

3. Browner WS, Newman B, Hulley SB: Estimating sample size and power, in Hulley SB (ed): Designing Clinical Research-An Epidemiologic Approach. Baltimore: Williams \& Wilkins, 1998, pp 55-83

4. Bujedo BM: Spinal opioid bioavailability in postoperative pain. Pain Pract 14:350-364, 2014

5. Crile GW: The kinetic theory of shock and its prevention through anoci-association (shockless operation). Lancet 182:7-16, 1913

6. Devin CJ, McGirt MJ: Best evidence in multimodal pain 
management in spine surgery and means of assessing postoperative pain and functional outcomes. J Clin Neurosci 22:930-938, 2015

7. Dickenson AH, Sullivan AF: Subcutaneous formalin-induced activity of dorsal horn neurones in the rat: differential response to an intrathecal opiate administered pre or post formalin. Pain 30:349-360, 1987

8. Goldberg DS, McGee SJ: Pain as a global public health priority. BMC Public Health 11:770, 2011

9. Harrison DM, Sinatra R, Morgese L, Chung JH: Epidural narcotic and patient-controlled analgesia for post-cesarean section pain relief. Anesthesiology 68:454-457, 1988

10. Hong R, Gauger V, Caird MS, Burke C: Narcotic-only epidural infusion for posterior spinal fusion patients: A singlecenter, retrospective review. J Pediatr Orthop 36:526-529, 2016

11. Johannes CB, Le TK, Zhou X, Johnston JA, Dworkin RH: The prevalence of chronic pain in United States adults: results of an Internet-based survey. J Pain 11:1230-1239, 2010

12. Jules-Elysee KM, Goon AK, Westrich GH, Padgett DE, Mayman DJ, Ranawat AS, et al: Patient-controlled epidural analgesia or multimodal pain regimen with periarticular injection after total hip arthroplasty: a randomized, double-blind, placebo-controlled study. J Bone Joint Surg Am 97:789-798, 2015

13. Kang H, Jung HJ, Lee JS, Yang JJ, Shin HY, Song KS: Early postoperative analgesic effects of a single epidural injection of ropivacaine administered preoperatively in posterior lumbar interbody spinal arthrodesis: a pilot randomized controlled trial. J Bone Joint Surg Am 95:393-399, 2013

14. Kissin I: Preemptive analgesia. Anesthesiology 93:11381143,2000

15. Lee BH, Park JO, Suk KS, Kim TH, Lee HM, Park MS, et al: Pre-emptive and multi-modal perioperative pain management may improve quality of life in patients undergoing spinal surgery. Pain Physician 16:E217-E226, 2013

16. Mhyre JM, Hong RW, Greenfield ML, Pace NL, Polley LS: The median local analgesic dose of intrathecal bupivacaine with hydromorphone for labour: a double-blind randomized controlled trial. Can J Anaesth 60:1061-1069, 2013

17. Møiniche S, Kehlet H, Dahl JB: A qualitative and quantitative systematic review of preemptive analgesia for postoperative pain relief: the role of timing of analgesia. Anesthesiology 96:725-741, 2002

18. Sekar C, Rajasekaran S, Kannan R, Reddy S, Shetty TA, Pithwa YK: Preemptive analgesia for postoperative pain relief in lumbosacral spine surgeries: a randomized controlled trial. Spine J 4:261-264, 2004

19. Siribumrungwong K, Cheewakidakarn J, Tangtrakulwanich $\mathrm{B}$, Nimmaanrat S: Comparing parecoxib and ketorolac as preemptive analgesia in patients undergoing posterior lumbar spinal fusion: a prospective randomized double-blinded placebo-controlled trial. BMC Musculoskelet Disord 16:59, 2015
20. Skinner HB: Multimodal acute pain management. Am J Orthop 33 (5 Suppl):5-9, 2004

21. Tripi PA, Kuestner ME, Poe-Kochert CS, Rubin K, Son-Hing JP, Thompson GH, et al: Intrathecal morphine attenuates acute opioid tolerance secondary to remifentanil infusions during spinal surgery in adolescents. J Pain Res 8:637-640, 2015

22. van Rappard JR, Tolenaar JL, Smits AB, Go PM: Spinal epidural abscess and meningitis following short-term epidural catheterisation for postoperative analgaesia. BMJ Case Rep 2015:210867, 2015

23. Woolf CJ: Evidence for a central component of post-injury pain hypersensitivity. Nature 306:686-688, 1983

24. Yukawa Y, Kato F, Ito K, Nakashima H, Machino M, Hirano $\mathrm{K}$, et al: A case-control study of preemptive analgesia for postoperative pain in patients undergoing posterior lumbar interbody fusion: continuous subcutaneous morphine alone and combined with intrathecal injection. J Spinal Disord Tech 23:333-337, 2010

25. Yukawa Y, Kato F, Ito K, Terashima T, Horie Y: A prospective randomized study of preemptive analgesia for postoperative pain in the patients undergoing posterior lumbar interbody fusion: continuous subcutaneous morphine, continuous epidural morphine, and diclofenac sodium. Spine (Phila Pa 1976) 30:2357-2361, 2005

\section{Disclosures}

The authors report no conflict of interest concerning the materials or methods used in this study or the findings specified in this paper.

\section{Author Contributions}

Conception and design: Aglio, Chi, Groff. Acquisition of data: Aglio, Abd-El-Barr, Orhurhu, Kim, Zhou, Crossley, Gosnell. Analysis and interpretation of data: Aglio. Drafting the article: Aglio, Abd-El-Barr. Critically revising the article: Aglio.

Reviewed submitted version of manuscript: Aglio, Orhurhu, Kim, Zhou, Gugino, Crossley, Gosnell, Chi, Groff. Approved the final version of the manuscript on behalf of all authors: Aglio. Statistical analysis: Abd-El-Barr. Study supervision: Aglio, Gugino. Patient care: Gosnell.

\section{Correspondence}

Linda S. Aglio: Brigham \& Women's Hospital, Harvard Medical School, Boston, MA. laglio@bwh.harvard.edu. 